\title{
Study on Maneuvering Target On-axis Tracking Algorithm of Modified Current Statistical Model
}

\author{
Xiong Zhenkai ${ }^{1,2}$, Li Fanying ${ }^{1}$, Zhang Lei $^{1}$ \\ ${ }^{1}$ China Shipbuilding Industry Corporation 713 Research Institute Zhengzhou, China \\ ${ }^{2}$ The School of Information Engineering of Zhengzhou UniversityZhengzhou, China
}

\begin{abstract}
Aiming at the model adaptability and the filter precision on the maneuvering target on-axis tracking, The paper put forward a filter algorithm based on modified current statistical model. The algorithm can enhance the model adaptability to the weak and non-maneuvering maneuvering target. The method uses Unscented Kalman Filter to obtain the importance density function of each particle, improves the Particle Filter estimation performance.By applying the proposed algorithm to the on-axis tracking system, the simulation results demonstrate that algorithm can effectively improve filter performance and tracking precision.
\end{abstract}

\section{Introduction}

Maneuvering target tracking technology is widely used in scientific research, aviation, aerospace and other fields, and put forward higher requirements. In particular, in the aspect of photoelectric tracking, due to the requirement of tracking precision, the requirements of maneuvering target tracking are higher. For this reason, the researchers propose methods such as composite axis tracking, equivalent composite tracking, on-axis tracking and multiple composite axis tracking. With the development of computer technology, on-axis tracking to solve the high precision optical tracking provides a good way[1]. One of the core of on-axis tracking is the filtering prediction algorithm, which is to predict the accurate target position information and velocity information for the control system[2,3,4,5]. The exact prediction premise of the maneuvering target prediction is that the target motion model can match the actual. However, because it is necessary to fully describe the actual state of the target motion, it is necessary to pass the high-order Taylor expansion, which is not desirable in the specific mathematical processing.

The establishment of the target model can not only facilitate the late mathematical calculations, but also similar to the target movement state. The "current" statistical model is a model that is more reasonable to describe the maneuvering target state[6,7]. The "current" probability density of the maneuvering acceleration is described by the modified Rayleigh distribution. The model is characterized by nonzero mean and modified Rayleigh distribution Maneuvering acceleration characteristics, and thus more realistic $[8,9,10,11,12,13]$. However, for weak and non-maneuvering maneuvering target, the current statistical model can not be fully described[14].

The key to the prediction of maneuvering target prediction is the measurement equation. The detection of sensor noise, the external uncertain disturbance and other nonlinear factors are the main aspects of the filter precision. The best way to solve the nonlinear factor is to use nonlinear filtering Prediction algorithm[15]. Based on these considerations, this paper designs an improved maneuvering target on-axis tracking filter algorithm for the current statistical model, which satisfies the fusion algorithm of Unscented Kalman Filter (UKF) and Particle Filter (PF) Nonlinear filter tracking requirements.

\section{On-axis Tracking Principle}

On-axis tracking is the target position information and speed information by computer technology to provide input for the servo system control. The servo system and the detection system are relatively independent[16,17]. The servo system is composed of speed loop and position loop. The detection system is composed of the detector and filter unit.After the detector output and the servo feedback information fusion, the parameter is used as filter input.Filter output is used as the servo system input[18].

The servo bandwidth should be wide for control precision and control optimization to reduce the detection noise interference[19,20,21,22], the detection system bandwidth should be narrow. On-axis tracking schematic diagram shown in Figure 1. 


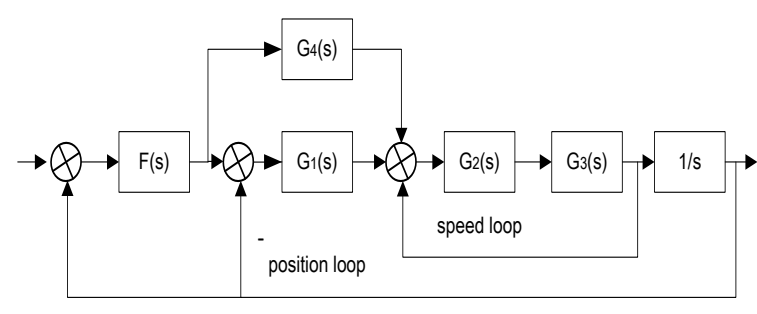

Figure 1. On-axis tracking principle figure.

In the figure, $\Delta \theta$ is the target error distance information by the sensor, $F(s)$ is the filtering predictive function, $G_{1}(s)$ is the position loop control function, $G_{2}(s)$ is the speed loop control function, $G_{3}(s)$ is the speed loop transfer function, and $G_{4}(s)$ is the feedforward control function.

\section{Modified Current Statistical Model}

The discrete state equation and the measurement equation of the one-dimensional current statistical model are:

$\mathrm{X}(k+1)=\mathrm{F}(k+1) \mathrm{X}(k)+\mathrm{G}(k) \bar{\omega}(k)+M(k)$

$\mathrm{Z}(k+1)=\mathrm{H}(k) \mathrm{X}(k)+\mathrm{N}(k)$

$\mathrm{X}(k)$ is System state vector,

$\mathrm{X}(k)=\left[\begin{array}{lll}x(k) & \dot{x}(k) & \ddot{x}(k)\end{array}\right]^{T}$,

$\mathrm{F}(k+1)$ is State transition matrix,

$F(k+1)=\left[\begin{array}{ccc}1 & T & \frac{e^{-\alpha T}+\alpha T-1}{\alpha^{2}} \\ 0 & 1 & \frac{1-e^{-\alpha T}}{\alpha} \\ 0 & 0 & e^{-\alpha T}\end{array}\right]$

$G(k)$ is input matrix,

$G(k)=\left[\begin{array}{c}\frac{1}{\alpha}\left(\frac{\alpha T^{2}}{2}-T+\frac{1-e^{-\alpha T}}{\alpha}\right) \\ T-\frac{1-e^{-\alpha T}}{\alpha} \\ 1-e^{-\alpha T}\end{array}\right]$

$\bar{\omega}(k)$ is the acceleration mean, $Z(k+1)$ is the measurement vector, $H(k)$ is the measurement matrix, $M(k)$ is the so-called noise, the covariance

$Q(k)=2 \alpha \delta_{a}^{2}\left[\begin{array}{lll}q_{11} & q_{12} & q_{13} \\ q_{21} & q_{22} & q_{23} \\ q_{31} & q_{32} & q_{33}\end{array}\right]$,

$R(k)$ is the covariance of the measurement noise $N(k)$.

$q_{11}=\frac{\left[1-e^{-2 \alpha T}+2 \alpha T-2 \alpha^{2} T^{2}+\frac{2 \alpha^{3} T^{3}}{3}-4 \alpha T e^{-\alpha T}\right]}{2 \alpha^{5}}$, $q_{12}=\frac{\left[e^{-2 \alpha T}+1-2 e^{-\alpha T}+2 \alpha T e^{-\alpha T}+\frac{2 \alpha^{3} T^{3}}{3}-2 \alpha T+\alpha^{2} T^{2}\right]}{2 \alpha^{4}}$,

$q_{13}=\frac{\left(1-e^{-2 \alpha T}-2 \alpha T e^{-\alpha T}\right)}{2 \alpha^{3}}$,

$q_{22}=\frac{\left(1+e^{-2 \alpha T}-2 e^{-\alpha T}\right)}{2 \alpha^{3}}$,

$q_{33}=\frac{\left(1-e^{-2 \alpha T}\right)}{2 \alpha}$

$\delta_{\omega}^{2}$ is the acceleration variance, $\alpha$ is the maneuvering frequency, $\mathrm{T}$ is the discrete time of the sampling period.

$\delta_{\omega}^{2}= \begin{cases}\frac{4-\pi}{\pi}\left(\omega_{\max }-\bar{\omega}_{k}\right)^{2} & \bar{\omega}_{k} \geq 0 \\ \frac{4-\pi}{\pi}\left(\omega_{-\max }+\bar{\omega}_{k}\right)^{2} & \bar{\omega}_{k}<0\end{cases}$

$\omega_{\max }$ is the maximum maneuvering acceleration, $\omega_{-\max }$ is the minimum maneuvering acceleration.

Due to the modified Rayleigh distribution, the target model $\frac{4-\pi}{4} \omega_{-\max } \leq \bar{\omega}_{k} \leq \frac{4-\pi}{4} \omega_{\max } \quad$ the acceleration distribution description is not available, thus resulting in poor precision for weak and nonmaneuvering tracking. Therefore, it is necessary to redescribe the acceleration variance[23,24]. In the Gaussian distribution, the probability of random variables beyond $3 \omega$ is 0.0027 , the acceleration mean is zero for the weak and non-maneuvering targets, and then,

$$
\begin{gathered}
\left|\frac{4-\pi}{4} \omega_{\max }\right| \leq 3 \delta_{\omega} \\
\delta_{\omega}^{2}=\frac{\left[(4-\pi) \omega_{\max } / 4\right]}{9}
\end{gathered}
$$

thus

$$
\delta_{\omega}^{2}=\left\{\begin{array}{cc}
\frac{4-\pi}{\pi}\left(\omega_{\max }-\bar{\omega}_{k}\right)^{2} & \bar{\omega}_{k}>\frac{4-\pi}{4} \omega_{\max } \\
\frac{4-\pi}{\pi}\left(\omega_{-\max }+\bar{\omega}_{k}\right)^{2} & \bar{\omega}_{k}<\frac{4-\pi}{4} \omega_{-\max } \\
\frac{\left.(4-\pi) \omega_{\max } / 4\right]}{9} & \frac{4-\pi}{4} \omega_{-\max } \leq \bar{\omega}_{k} \leq \frac{4-\pi}{4} \omega_{\max }
\end{array}\right.
$$

\section{PF and UKF fusion filter}

UKF through Unscented transformation, the calculation sampling point through the non-linear propagation, its posterior statistical value use linear regression to obtain posterior statistics, the Gaussian random variable Taylor 
expansion to the third order, is a filter precision higher nonlinear filter[25].

$\mathrm{PF}$ is a posterior probability density based on Monte Carlo random sampling particle weight and deapproximation states[26]. It is very suitable for nonlinear filtering due to the sample form rather than function form. However, since the particle filter usually uses the state transition density as an important density function, the filter is liable to fail when the new measurement present to the tail of prior distribution or is found to be more sharp with respect to the prior distribution. At the same time, The repeat sampling link, so that the diversity of particles lost, filter performance decreased.

Therefore, it is necessary to generate the importance density function by a suboptimal filtering method. For this reason, UKF is used to generate the importance density function, and the latest measurement information is used in the particle sampling process to improve the sampling particle validity of approximation the distribution[27].

The specific steps of the UKF and PF fusion filter:

(1)Initialization: $\mathrm{k}=0$, random sampling points are drawn from prior distributions .

$\hbar_{0}^{i}, \hbar_{0}^{i} \sim p\left(\hbar_{0}\right)(i=1,2, \cdots N)$;

$\omega_{0}^{i}=\frac{1}{n}$ is the weight, $\mathrm{n}$ is the particle number.

(2) Importance sampling: for each particle $\hbar_{k-1}^{i}$, the mean $\bar{\hbar}_{k}^{i}$ and covariance $P_{k}^{i}$ is calculated by UKF, and get the importance density function $N\left(\bar{\hbar}_{k}^{i}, P_{k}^{i}\right)$ [28].

$$
q\left(\hbar_{k}^{i} \mid \hbar_{k-1}^{i}, \mathrm{z}_{k}\right)=N\left(\bar{\hbar}_{k}^{i}, P_{k}^{i}\right)
$$
weights

(3) Weight update: extract $\mathrm{N}$ particles, calculate

$$
\omega_{k}^{i}=\omega_{k-1}^{i} \frac{p\left(\mathrm{z}_{k} \mid \hbar_{k}^{i}\right) p\left(\hbar_{k}^{i} \mid \hbar_{k-1}^{i}\right)}{q\left(\hbar_{k}^{i} \mid \hbar_{k-1}^{i}, \mathrm{z}_{k}\right)}
$$

normalizing weights

$$
\bar{\omega}_{k}^{i}=\omega_{k}^{i} / \sum_{i=1}^{n} \omega_{k}^{i}
$$

(4) Resampling[29]:

Resampling judgment: when

$$
1 / \sum_{i=1}^{n} \omega_{i}^{2}>N_{o}
$$

nonresampling; otherwise resampling, $N_{o}$ is the sampling threshold .

(a)Rank $\mathrm{n}$ particles by weight, the former $n_{1}$ particle were selected and normalized by equation (9).

(b) Calculate the filter density for selected particles

$$
p\left(\hbar_{k} \mid z_{k}\right)=\sum_{i=1}^{n_{1}} \omega_{k}^{i} \delta\left(\hbar_{k}-\hbar_{k}^{i}\right)
$$

(c) The weight of the selected $n_{1}$ particle are restored to the pre-normalized weight by equation (9).

(d) All $\mathrm{n}$ particles are normalized by equation (9).

(5)Estimate calculate:

$$
\begin{gathered}
\hat{\hbar}_{k}=\sum_{i=0}^{n} \omega_{k}^{i} \hbar_{k}^{i *} \\
P_{k}=\sum_{i=0}^{2 n} W_{i}^{c}\left[\chi_{k}-\hat{\hbar}_{k}\right]\left[\chi_{k}-\hat{\hbar}_{k}\right]^{T}
\end{gathered}
$$

$W_{i}^{c}$ is the sigma weight, $\chi_{k}$ is the sample point.

\section{(6)Jump calculating}

$\mathrm{K}=\mathrm{k}+1$,go to step 2 .

\section{Simulation and Experiment analysis}

Aiming at the TV tracking system, the system simulation model is established. transfer function

$$
\begin{aligned}
& G_{1}(s)=\frac{37.5(0.01 s+1)}{\left(0.34 s^{2}+0.05 s+1\right)} \\
& G_{2}(s) G_{3}(s)=\frac{37.5}{(0.01 s+1)\left(0.34 s^{2}+0.05 s+1\right)}
\end{aligned}
$$

Based on the origin of the coordinates, if the target model is constant velocity model,the equation (1) and (2) can be showed:

$$
\begin{aligned}
& \mathrm{X}(k+1)=\left[\begin{array}{cccc}
1 & T & 0 & 0 \\
0 & 1 & 0 & 0 \\
0 & 0 & 1 & T \\
0 & 0 & & 1
\end{array}\right] \mathrm{X}(k)+\left[\begin{array}{c}
T^{2} / 2 \\
T \\
T^{2} / 2 \\
T
\end{array}\right] M(k) \\
& \mathrm{Z}(k+1)=\left[\begin{array}{c}
\sqrt{\left(x_{k}^{2}+y_{k}^{2}\right)^{2}} \\
a c \tan \frac{y_{k}}{x_{k}}
\end{array}\right]+\mathrm{N}(k) \\
& \mathrm{X}(k) \text { is System state vector, } \\
& \mathrm{X}(k)=\left[\begin{array}{llll}
x_{k} & v_{x_{k}} & y_{k} & v_{y_{k}}
\end{array}\right]^{T},
\end{aligned}
$$

it show the displacement and velocity components in $\mathrm{x}$ and $\mathrm{y}$ directions. $M(k)$ is the gaussian white noise, the covariance $Q(k)=\left[\begin{array}{cc}0.5^{2} & 0 \\ 0 & 0.5^{2}\end{array}\right] . N(k)$ is also the gaussian white noise, the covariance

$$
R(k)=\left[\begin{array}{cc}
20^{2} & 0 \\
0 & 0.05^{2}
\end{array}\right]
$$

The period of filtering $T=0.1 s$, the initial value $\mathrm{X}(0)=\left[\begin{array}{llll}1000 & 150 & 1000 & 30\end{array}\right]^{T}$, the particle number is 100. One hundred Monte Carlo simulation is performed by Intel core i7-5500U computer. The simulation applied the MCS-PF、MCS-UKF and MCS-UPF. Position error curve shown in Figure2,velocity error curve shown in Figure3, single performance period shown in table1. 


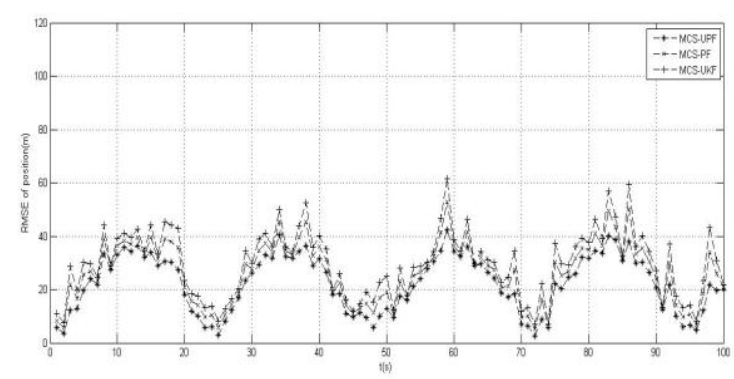

Figure 2. Position error curve figure.

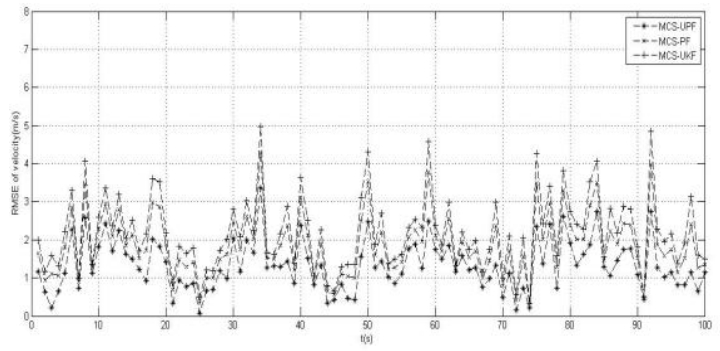

Figure 3. Velocity error curve figure.

Table 1. Time comparision.

\begin{tabular}{|c|c|}
\hline Filter method & Single time(s) \\
\hline MCS-UKF & 0.65 \\
\hline MCS-PF & 1.43 \\
\hline MCS-UPF & 3.19 \\
\hline
\end{tabular}

\section{Conclusions}

Aiming at the problem of poor adaptability for filtering models and the problem of low filter precision, the paper put forward a filter algorithm based on modified current statistical model for improving the precision of on-axis tracking system.The algorithm enhances the model's adaptive ability to weak and non-maneuvering maneuvering target.The algorithm increases the estimated capacity of PF algorithm by UKF to calculate the importance density function. The algorithm is applied to the on-axis tracking system simulation. It can effectively improve the filtering performance, enhance the tracking precision and stability. The algorithm improves the filtering performance, but increases the computational complexity. The next step study must focus on the problem to reduce calculation and shorten the calculation time.

\section{References}

1. Michanil N P. Signal Processing.87,7(2007)

2. O.Cappe, S.Godsill, E.Moulines.Proceedings of IEEE. 95 ,5(2007)

3. Z K Xiong,F Y Li..2017 IEEE 2nd Advanced Information Technology, Electronic and Automation Control Conference(2017)
4. Lu Y,Wu T,Chun Zhu S. Proceedings of the IEEE Conference on Computer Vision and Pattern Recognition(2014)

5. Merwe v.d.R, Douce A.Cambridge University Enginnering Department, England Cambridge University press(2000)

6. J.Simon, K. U. Jeffrey. Proceedings of IEEE.92, 3(2004)

7. $\mathrm{H}$ R ZHOU.Acta Aeronautica Et Astronautuca Sinica.4,1(1983)

8. Y Qing, L Ming.2017 International Conference on Smart Grid and Electrical Automation(2017)

9. Z K XIONG, T F CHEN. High Power Laser and Particle Beams.24,6 (2012)

10. $\mathrm{X}$ Wang, $\mathrm{Z}$ Q Hou. IEEE Transactions on Cybernetics, 99(2017)

11. X J FANG, F LIU, Y QIN. Acta Electronica Sinica. 34, 6 (2006)

12. Nadine M. Moacdieh, Nadine Sarter.IEEE Transactions on Human-Machine Systems.47,6(2017)

13. J LIU, Y J WANG, J ZHANG. IEEE Conf on Decision and Control (2014)

14. F H Liu, $\mathrm{C}$ Gong, $\mathrm{T}$ Zhou,K Fu.IEEE Transactions on Multimedia. 19,12(2017)

15. Y SHI, C Z HAN. Journal of Acta Automatica Sinica. 37(2011)

16. Seon Heo,Hyung Il Koo,Nam Ik Cho. IEEE Transactions on Circuits and Systems for Video Technology.27,11(2017)

17. $\mathrm{H} X \mathrm{YU}, \mathrm{C} \mathrm{K}$ FU. Proc. of the 2nd International conference on Signal Processing Systems(2010)

18. $\mathrm{T} F \mathrm{Fu}, \mathrm{Y} \mathrm{Lu}, \mathrm{S} \mathrm{C}$ Zhu. IEEE Transactions on Pattern Analysis and Machine Intelligence.39, 12 (2017)

19. Pavani S K,Delgado D,Frangi A F. Pattern Recognition.43,1(2010)

20. N Wang, S Lv; Meng Joo Er, W H Chen. IEEE Transactions on Intelligent Vehicles. 1, 3( 2017)

21. Danelljan M,Hager G,Khan F.Proceedings of the Conference on British Machine Vision Conference(2014)

22. X D Zhao, $P$ Shi. IEEE Transactions on Neural Networks and Learning Systems.27, 9(2016)

23. K Mechrotra, P R Mahapatra. IEEE Trans. On Aerospace and Electronic Systems.33, 4(1997)

24. Lasitha Mekkayil; Hariharan Ramasangu. 2017 IEEE Region 10 Symposium(2017)

25. $\mathrm{J} G \mathrm{GA}, \mathrm{T}$ TANG. Infrared and Laser Engineering.42, 1(2013)

26. Ma C,Huang J B,Yang X. Proceeding of the IEEE International Conference on Computer on Computer Vision(2015)

27. Lin M,Zhang C,Zhang Z. Neurocomputing.159(2015)

28. $G \mathrm{G}$ Rigatos. IEEE Transactions on Industrial Electronics.59, 10(2012)

29. Cehovin L,Kristan M, Leonardis A. IEEE transactions on pattern analysis and machine intelligence.35,4(2015) 